\title{
Glycemic control and survival of patients with coexisting diabetes mellitus and gastric or esophageal cancer
}

\section{Fut}

\author{
Nina J Karlin*,1, Matthew R Buras², Heidi E Kosiorek², Patricia M Verona ${ }^{3}$ \& Curtiss B Cook ${ }^{4}$ \\ ${ }^{1}$ Division of Hematology \& Medical Oncology, Department of Internal Medicine, Mayo Clinic Hospital, Phoenix, AZ 85054, USA \\ ${ }^{2}$ Division of Endocrinology, Department of Biostatistics, Mayo Clinic, Scottsdale, AZ 85259, USA \\ ${ }^{3}$ Department of Information Technology, Department of Biostatistics, Mayo Clinic Hospital, Phoenix, AZ 85054, USA \\ ${ }^{4}$ Department of Internal Medicine, Division of Endocrinology, Mayo Clinic, Scottsdale, AZ 85259, USA \\ *Author for correspondence: karlin.nina@mayo.edu
}

\begin{abstract}
Aim: To examine effects of diabetes mellitus (DM) on survival in gastric or esophageal (GE) cancer and the cancers' effects on glycemic control. Materials \& methods: Patients with GE cancer with and without DM were matched 1 to 1 (2006-2016). Characteristics were compared and survival assessed with Kaplan-Meier method and Cox regression. Mixed models compared hemoglobin $A_{1 c}$ and glucose over time. Results: Among DM cases, mean hemoglobin $A_{1 c}$ was $6.8 \%$ in the year after cancer diagnosis. Three-year overall survival was $46 \%$ with DM versus $52 \%$ without DM (hazard ratio [95\% Cl]: 1.95 [1.14-3.34]; $p=0.02$ ). Conclusion: GE cancer and its treatment did not affect glycemic control. Risks of death and progression were greater for patients with DM than patients without DM.

Lay abstract: The objective of this study was to identify the effect of diabetes mellitus (DM) on survival of patients with gastric or esophageal (GE) cancer and to determine whether GE cancer and its treatment affected glycemic control. We used an institutional cancer registry to identify 184 patients with GE cancer and grouped them by the presence $(n=92)$ or absence $(n=92)$ of DM. Patients were matched by age and year of cancer diagnosis. GE cancer did not affect glycemic control, and risks of death and disease progression were greater in patients with DM than patients without DM.
\end{abstract}

First draft submitted: 20 March 2019; Accepted for publication: 1 May 2019; Published online: 26 June 2019

Keywords: cancer $\bullet$ endocrinology $\bullet$ glycemia $\bullet$ malignancy $\bullet$ mortality $\bullet$ outcomes research

Although the incidence of gastric cancer has decreased over the past decade, approximately 26,240 cases will have been diagnosed in 2018 in the USA, and approximately 10,800 people are projected to die as a consequence [1]. The incidence of esophageal cancer has also decreased slightly over the past decade. Nonetheless, 17,290 new cases of esophageal cancer will have been diagnosed in the USA in 2018, and 15,850 people were expected to die of it [1]. Adenocarcinoma accounts for most of the esophageal cancers diagnosed in the USA [2]. Obesity is a risk factor for esophageal adenocarcinoma and may be a risk factor for gastric cancer [3,4]. Studies are inconclusive about whether diabetes mellitus (DM) is associated with an increased risk of gastric cancer [5,6].

In some studies, DM has been associated with an increased risk of esophageal cancer [7,8]. However, other data suggest that DM is not associated with a higher incidence of gastric cancer and that the incidence of esophageal cancer is lower for patients with DM [9]. Nonetheless, shared risk factors (e.g., obesity, inflammation, hyperinsulinemia, insulin resistance) exist between these two cancers and DM, making it plausible that the latter could in some way influence the incidence or outcomes, or both, of gastric or esophageal (GE) cancers $[7,10]$.

In a series of separate analyses, the authors previously examined the interaction between different solid tumors (breast, prostate, lung, colorectal and pancreas) [11-15] with DM. Unlike other reports in the literature that examined how DM affects survival for cancer patients, the authors used a matched case-control methodology where cancer patients without DM (controls) were compared with those with DM (cases). A common result thus far has been that DM did not affect survival in these cancers and that the presence of the cancer did not worsen glycemic control. 
Given the different pathophysiologic characteristics and behavior of the different cancer types, continued effort is important to extend these analyses to other types of solid organ cancers. Hence, comprehensive data for GE cancers and DM variables were gathered to investigate whether DM had affected survival of patients with GE cancers and to analyze whether GE cancers and their treatments had affected glycemic control. This paper was presented at the ASCO GI Cancer Symposium, CA, USA, January 17-19, 2019, and published in abstract form in: J. Clin. Oncol. (2019); 37 (4 Suppl.) 73.

\section{Methods}

Case selection was similar to that described in the authors' previous studies [11-15]. Briefly, institutional review board approval was obtained for the present retrospective case-control study. Electronic health records (EHRs) of patients with GE cancer newly diagnosed from 1 January 2006 to 31 December 2016 were obtained from the institutional cancer registry. In addition to demographic data, the registry contained the date of cancer diagnosis and the tumor stage. This initial data file was linked to EHRs to determine which patients had a diagnosis of DM during the study period. The authors excluded patients who received full or partial cancer treatment at another institution or had another cancer preceding their GE cancer diagnosis. As previously described, patients with GE cancer and DM were matched (1 to 1 with use of the Greedy algorithm [16]) to patients with GE cancer but no DM. Variables included in the matching algorithm were age, sex and year of GE cancer diagnosis.

Glucose and hemoglobin $\mathrm{A}_{1 \mathrm{c}}\left(\mathrm{HbA}_{1 \mathrm{c}}\right)$ values were derived from the institution's laboratory information system. The EHRs were then reviewed for the following additional detailed information: type of GE cancer treatment (e.g., surgical procedure, chemotherapy, radiation, targeted therapy) and data related to DM (e.g., date of DM diagnosis, type of DM therapy, DM complications).

\section{Statistical analysis}

The statistical analyses were similar to those used for the authors' previous studies [11-15]. Patient demographic and clinical characteristics were compared among patients who had GE cancer with or without DM. Continuous variables were compared with use of paired t-tests; categorical variables, with the McNemar test or Bowker test for symmetry. $\mathrm{HbA}_{1 \mathrm{c}}$ levels during the first year after $\mathrm{GE}$ cancer diagnosis were evaluated with a linear mixed model in $\mathrm{DM}$ cases only $\left(\mathrm{HbA}_{1 \mathrm{c}}\right.$ values were unavailable for most patients without $\left.\mathrm{DM}\right)$. A fixed effect for time (days) and a patient-specific random effect were included. A similar approach was used for modeling glucose values during that year. Fixed effects included days, designation of case or control, an interaction term (days $\times$ case-control designation) and patient-specific and matched pair-specific random effects. Optimal glycemic control was defined as a mean glucose value less than $126 \mathrm{mg} / \mathrm{dl}$.

Overall survival (OS) was defined as the time from GE cancer diagnosis until death due to any cause. For OS, patients were considered censored at the last known follow-up date when death was not documented in the EHR. Progression-free survival (PFS) was defined as the time from GE cancer diagnosis until disease progression or death of any cause. Patients were considered censored at the last known date when they were alive if disease progression or death had not occurred. Three-year OS and PFS were estimated with the Kaplan-Meier method. A Cox proportional hazards regression model was used with the matched pairs as the strata variable. Sample size was based on the number of available cases from 2006 through 2016; it provided 80\% power to detect a difference in hazard ratio of 2.2 or greater for OS. The $\mathrm{p}<0.05$ was considered statistically significant.

Statistical software (SAS version 9.4; SAS Institute Inc.) was used for analysis. Data are expressed as number (\%) for categorical variables and as mean (SD) for continuous variables.

\section{Results}

Patient characteristics

In total, 92 matched pairs of patients $(\mathrm{N}=184)$ were analyzed. Mean age of the entire cohort was 68 years, $91 \%$ were white, $78 \%$ were men and $53 \%$ had stage III or IV disease (Table 1). Most patients were married (80\%), had a history of smoking (current, 12\%; former, 62\%), were retired (51\%) and had a European Cooperative Oncology Group score of $1(75 \%)$. BMI was significantly different between the DM group and the non-DM group ( $\mathrm{p}=$ 0.006). Alcohol use at the time of cancer diagnosis was more prevalent in patients without DM than those with $\mathrm{DM}(\mathrm{p}=0.02)$. No differences were detected between any other variable for the two groups.

Nearly all the patients with DM had their DM diagnoses before the GE cancer diagnoses (Table 2). In those instances where it was documented, the mean (SD) self-reported duration of DM diagnosis was 10.9 (7.40) years. 
Table 1. Demographic characteristics related to gastric or esophageal cancer and diabetes mellitus status.

\section{Characteristic $^{\dagger}$}

Age at cancer diagnosis, mean year (SD)

Age at cancer diagnosis, mean year (SD)

Male sex

Race:

- White

- Non-white

Tumor stage:

$-1$

- II

- III

- IV

- Missing data

Cancer location:

- Gastric

- Esophageal

- Missing data

Cancer type:

- Adenocarcinoma

- Squamous cell carcinoma

- Other

- Missing data

- BMI

- Patients ( $n$ )

- Mean (SD)

Marital status at cancer diagnosis:

\section{- Married}

- Not married

Alcohol use at cancer diagnosis:

- Yes

- No

- Unknown

Smoking status at cancer diagnosis:

- Never

- Former

- Current

Employment status at cancer diagnosis:

- Employed

- Not employed

- Missing data

ECOG score at cancer diagnosis:

$-0$

$-1$

$-2$

$-3$

$-4$

Use of corticosteroids:

-Yes $46(52)$

No

- Missing data

(2)

\begin{tabular}{l} 
No \\
68 \\
72 \\
\hline 86 \\
6 \\
\hline
\end{tabular}

No $(n=92)$

68 (11.06)

$72(78)$

$86(94)$

$6(6)$

$33(38)$

$10(12)$

29 (34)

14 (16)

6

$23(25)$
$68(75)$
1

1

$70(77)$
$7(8)$

$14(15)$

1

86

86

$27.2(5.06)$

73 (79)

19 (21)

$60(65)$

29 (32)

3 (3)

19 (21)

57 (62)

16 (17)

17 (19)

73 (81)

2

2

12 (13)

73 (79)

$6(7)$

1 (1)

$0(0)$

46 (52)

43 (48)

3
35 (38)

$29.7(5.53)$

1

$\dagger$ Values are presented as number and percentage of patients unless specified otherwise.

$\ddagger$ McNemar test or Bowker test for symmetry.

$\S$ Paired-sample t-test.

BMI: Body mass index; DM: Diabetes mellitus; ECOG: European Cooperative Oncology Group; SD: Standard deviation.
DM

Yes $(\mathrm{n}=92)$

68 (11.12)

72 (78)

82 (89)

10 (11)

24 (27)

15 (17)

30 (34)

$19(22)$

4

57 (62)

0

$74(80)$

2 (2)

16 (17)

0

83

83

75 (82)

17 (19)

47 (51)

45 (49)

0 (0)

29 (32)

57 (62)

6 (7)

$23(25)$

68 (75)

13 (14)

65 (71)

9 (10)

4 (4)

1 (1)

p-value

Total $(n=184)$

68 (11.06) Matched

144 (78) Matched

$0.58^{\ddagger}$

$168(91)$

$16(9)$

$0.18^{\ddagger}$

57 (33)

25 (14)

59 (34)

33 (19)

10

$0.06^{\ddagger}$

58 (32)

125 (68)

1

$0.22^{\ddagger}$

144 (79)

9 (5)

30 (16)

1

$0.006^{\S}$

169

$28.4(5.42)$

$0.66^{\ddagger}$

$148(80)$

$36(20)$

$107(58)$

74 (40)

3 (2)

$0.10^{\ddagger}$

48 (26)

$114(62)$

$22(12)$

$0.26^{\ddagger}$

40 (22)

141 (78)

3

$0.42^{\ddagger}$

25 (14)

$138(75)$

15 (8)

5 (3)

1 (1)

$49(54) \quad 95(53)$

42 (46)

85 (47)

1

4

$0.02^{\ddagger}$


Table 2. Diabetes mellitus therapy for the 92 patients with gastric or esophageal cancer.

\begin{tabular}{|c|c|c|}
\hline Characteristic & DM & Patients ( $n$ ) \\
\hline \multicolumn{3}{|c|}{ DM diagnosis preceded GE cancer diagnosis of patients with documentation, $n(\%)$ : } \\
\hline - Yes & $81(98)$ & 83 \\
\hline- No & $2(2)$ & \\
\hline \multicolumn{3}{|c|}{ Time since DM diagnosis if preceded cancer diagnosis (year): } \\
\hline - Mean (SD) & $10.9(7.40)$ & 47 \\
\hline \multicolumn{3}{|c|}{ DM therapy, $n(\%):$} \\
\hline - Diet & $17(21)$ & 84 \\
\hline - Oral & $41(48)$ & \\
\hline - Insulin & $18(22)$ & \\
\hline - Oral + insulin & $7(8)$ & \\
\hline - Other & $1(1)$ & \\
\hline - Missing data & 8 & \\
\hline \multicolumn{3}{|c|}{ Method of DM therapy changed within 1 year after cancer diagnosis, $n(\%)$ : } \\
\hline -Yes & $20(24)$ & 92 \\
\hline- No & $49(60)$ & \\
\hline - Unknown & $13(16)$ & \\
\hline - Missing data & 10 & \\
\hline
\end{tabular}

Table 3. Comparison of gastric and esophageal cancer therapy for patients with diabetes mellitus and those without it.

\begin{tabular}{|c|c|c|c|c|}
\hline \multirow[t]{2}{*}{ Therapy } & \multicolumn{3}{|c|}{ DM, n (\%) } & \multirow[t]{2}{*}{ p-value ${ }^{\dagger}$} \\
\hline & Number $(\mathrm{n}=92)$ & Yes $(n=92)$ & Total $(n=184)$ & \\
\hline Chemotherapy: & & & & 0.71 \\
\hline - Yes & $46(57)$ & $49(57)$ & $95(57)$ & \\
\hline - No & $35(43)$ & $37(43)$ & $72(43)$ & \\
\hline - Missing data & 11 & 6 & 17 & \\
\hline Immunotherapy: & & & & 0.36 \\
\hline- No & $80(87)$ & $85(92)$ & $165(90)$ & \\
\hline - Unknown & $12(13)$ & $7(8)$ & $19(10)$ & \\
\hline Targeted & & & & 0.56 \\
\hline - Yes & $1(1)$ & $3(4)$ & $4(2)$ & \\
\hline - No & 79 (99) & $82(96)$ & $161(98)$ & \\
\hline - Missing data & 12 & 7 & 19 & \\
\hline Radiation: & & & & 0.69 \\
\hline - Yes & $36(44)$ & $35(41)$ & $71(43)$ & \\
\hline- No & $45(56)$ & $51(59)$ & $96(57)$ & \\
\hline - Missing data & 11 & 6 & 17 & \\
\hline
\end{tabular}

Some patients $(41 / 84,48 \%)$ were receiving oral DM medications at the time of their GE cancer diagnosis. Of the 84 patients with DM, 25 (30\%) were taking insulin either alone or in combination with oral agents. Only 20 patients $(24 \%)$ changed their DM therapy within 1 year after the cancer diagnosis: eight patients switched to insulin treatment, six to diet, four to oral medicine and insulin and two to oral medication. Insulin use was at 32\% within 1 year after the cancer diagnosis. Corticosteroids were taken by $54 \%$ of patients with DM and $52 \%$ without DM.

DM complications were documented for only $16(20 \%)$ of patients before cancer diagnosis. This percentage was stable within the first year after cancer diagnosis. Among patients with GE cancer and documentation, 95 (57\%) received chemotherapy and 71 (43\%) received radiation therapy (Table 3$)$. 


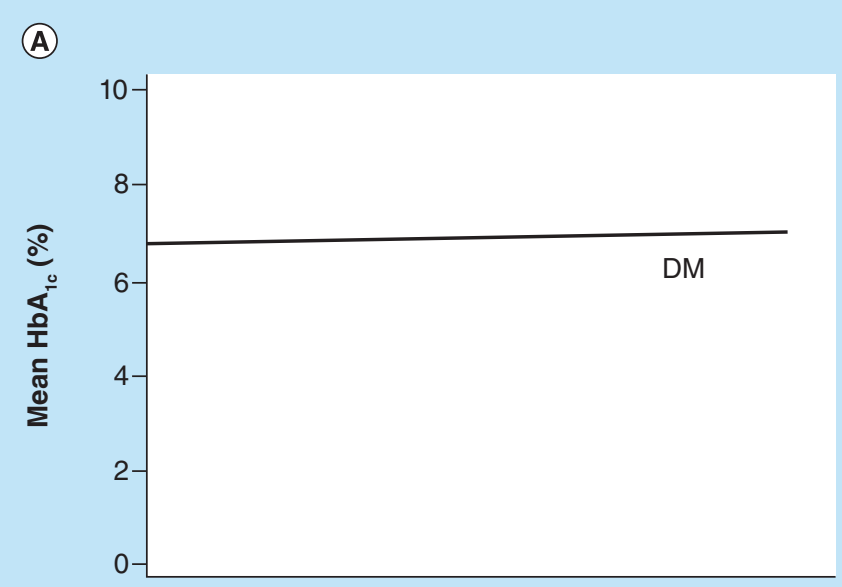

(B)

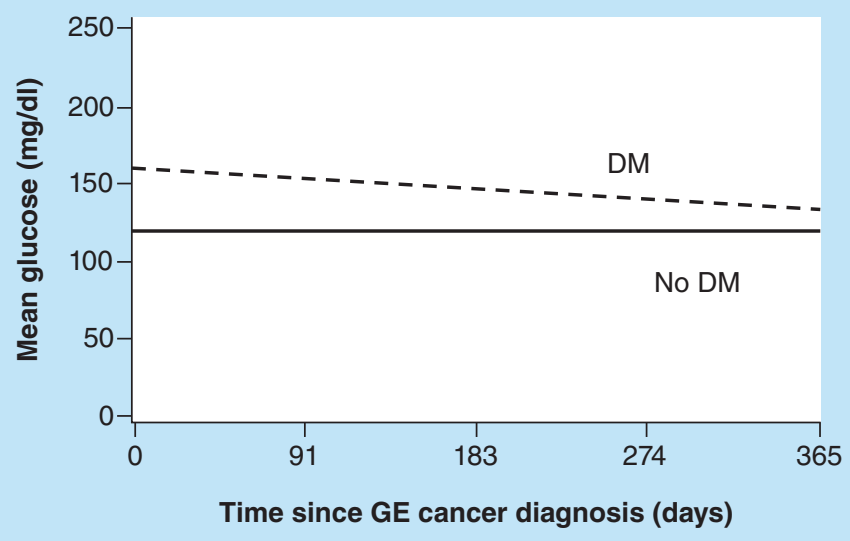

Figure 1. Values measured over the first year after gastric or esophageal cancer diagnosis. (A) $\mathrm{Hb}_{1 \mathrm{c}}$ percentages of patients with DM. (B) Comparison of glucose concentrations of patients with DM vs no DM.

DM: Diabetes mellitus; GE: Gastric or esophageal; $\mathrm{HbA}_{1 \mathrm{c}}$ : Hemoglobin $\mathrm{A}_{1 \mathrm{c}}$.

\section{GE cancer effect on DM \& metabolic control}

The $\mathrm{HbA}_{1 \mathrm{c}}$ data measured within 1 year after GE cancer diagnosis were available for 50 patients with DM (Figure 1A). For these patients, the mean (SD) $\mathrm{HbA}_{1 \mathrm{c}}$ level was 6.8\% (1.3\%), and 20 (40\%) had at least one $\mathrm{HbA}_{1 \mathrm{c}}$ measurement of $7.0 \%$ or greater. Mean glucose was significantly different between the DM and non-DM groups $(149 \mathrm{mg} / \mathrm{dl}$ vs $116 \mathrm{mg} / \mathrm{dl} ; \mathrm{p}<0.001)$. For glucose, a significant interaction effect $(\mathrm{p}=0.005)$ was observed as patients with DM showed a decrease in glucose values over time compared with patients without DM (Figure 1B).

\section{DM effect on GE cancer survival}

Three-year (95\% CI) OS (median follow-up, 35 months) was estimated at 46\% (36-58\%) for patients with DM versus 52\% (41-64\%) for those without DM (Figure 2). Three-year (95\% CI) PFS was estimated at 40\% (31-53\%) for patients with DM compared with 50\% (40-63\%) for those without it (Figure 3). Hazard ratio (using stratification for matched pairs) for OS was 1.95 (95\% CI: 1.14-3.34; $\mathrm{p}=0.02$ ); for PFS, 1.74 (95\% CI: 1.04-2.90; $\mathrm{p}=0.03)$.

\section{Discussion}

More data regarding individual patient outcomes are needed on the interaction of DM and solid cancerous tumors. The authors previously have investigated the effects of several different solid tumors - breast, prostate, lung, colorectal and pancreatic tumors - and DM on patient outcomes [11-15]. In all these matched case-control studies, DM did not affect survival of patients (typically monitored for nearly 5 years), and the cancer did not affect 


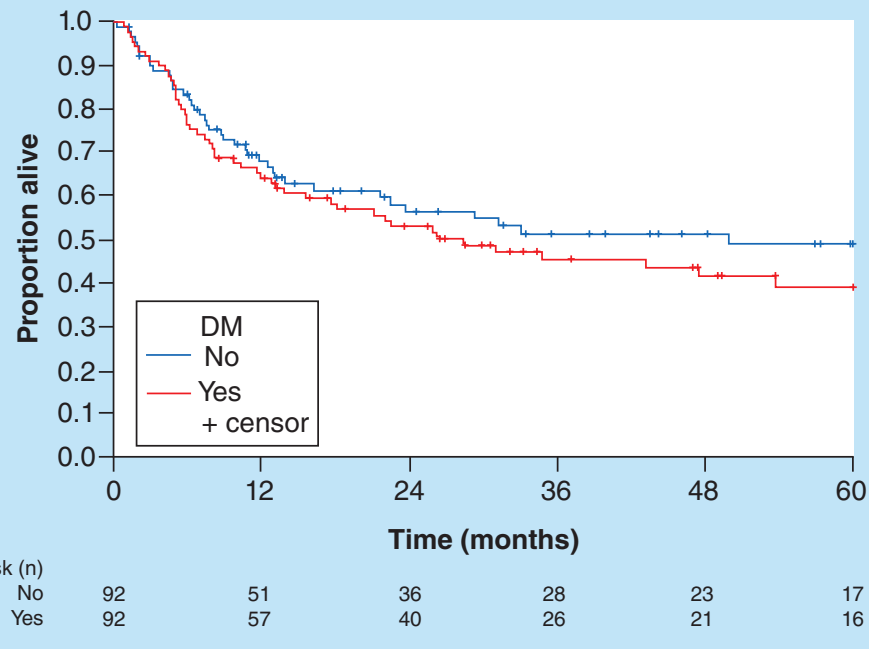

Figure 2. Overall survival of patients with and without diabetes mellitus. DM: Diabetes mellitus.

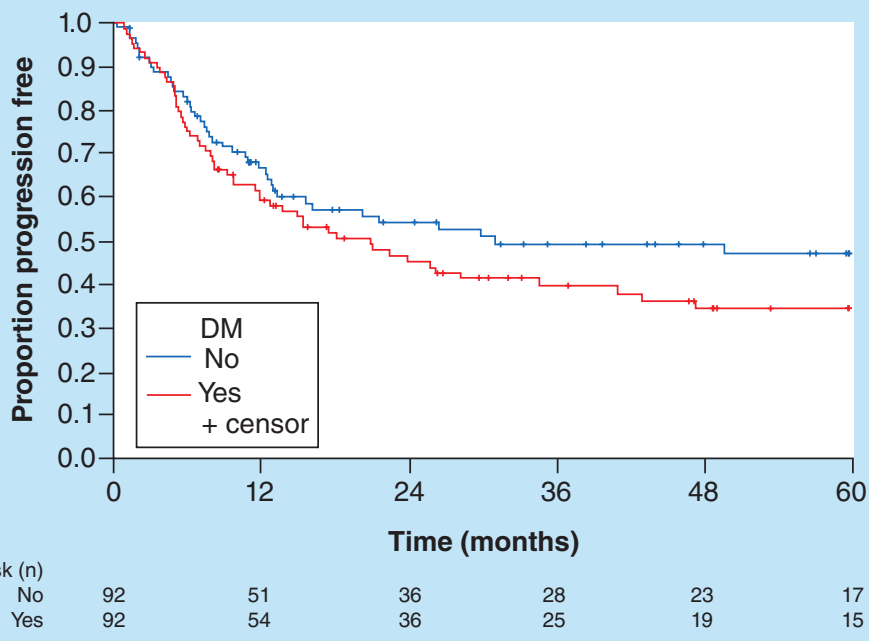

Figure 3. Progression-free survival of patients with and without diabetes mellitus. DM: Diabetes mellitus.

glycemic control in patients with DM over a 1-year period after a cancer diagnosis. The results of the present study show that GE cancer does not affect glycemic control negatively, but that risks of death and disease progression are greater for patients with DM than patients without DM.

Patients with gastric cancer and DM have a high mortality risk [17]. An even higher mortality risk has been reported in cases of gastric cancer with metabolic syndrome, especially hyperglycemia [18]. Some data suggest that a preoperative elevated glucose level corresponds with poor survival for patients with esophageal squamous cell cancer [19]. Certain comorbidities, such as atrial fibrillation and hypothyroidism, have affected esophageal cancer survival [20]. Among patients with esophagectomy for squamous cell cancer, OS is shorter for patients with DM and weight loss [21].

Unlike the authors' prior analyses, the risks of death and disease progression in the present case-control study were greater for patients with DM than patients without it. Nutritional compromise and diminished immunocompetence in patients with DM may be contributing factors to these findings. In addition, an undiscovered biomarker or 
metabolic factor may selectively alter the mortality rate in DM versus no DM. Furthermore, patients with DM and GE cancer may have more DM complications. Given that DM complications are not documented consistently, the DM complication rate may have been underestimated in this study. Finally, it is also possible that patients with GE cancer and DM received less aggressive therapy from their treating physicians. This is a phenomenon recognized in the literature [22].

Typically, GE cancers are lumped together because of close anatomical proximity and historical precedence. However, GE cancers have distinctive histologic features and molecular footprints. A comparative molecular analysis of esophageal squamous cell cancer, esophageal adenocarcinoma and gastric adenocarcinoma through next-generation sequencing has shown that each tumor has dissimilar expression of genes [23]. A larger sample size would be needed to determine whether expression of these genes is different in GE cancers with and without associated DM. Nonetheless, GE tumors in patients with DM may have a greater mutational load and a more aggressive molecular phenotype. This may be due to a milieu of chronic inflammation and the relative proportion and absolute quantity of hormones found in patients with DM (e.g., vasoactive intestinal peptide, glucagon-like peptide, insulin) that are active in the stomach and esophagus.

Our study has limitations. It is retrospective in nature, and its findings should be confirmed in a larger cohort over a longer period. Most patients were white, and thus results may be less applicable to patients of other ethnic and racial backgrounds. Finally, the cause of death was not available in the EHRs. Glycemic control was assessed at 1 year only, and therefore, would not account for any transient worsening that might have occurred during that 12-month period.

Results reporting that GE cancer does not affect glycemic control, at least at 12 months of follow-up, should be reassuring to practicing providers. However, future measures should be taken to explore and improve the increased risks of death and disease progression seen in patients with DM. These investigations could include medications used for DM or markers of immune function in patients with both DM and these cancers. Perhaps patients with $\mathrm{DM}$ and GE cancer would benefit from the more aggressive nutritional input along their treatment trajectory. At last, patients with DM and GE cancer might benefit from different systemic therapy regimens that concentrate more on checkpoint inhibitor therapy or targeted therapy (such as therapy based on vascular endothelial growth factor inhibitor) than from conventional cytotoxic chemotherapy.

\section{Future perspective}

These data add to the literature regarding the relationship between DM, cancer, death and glycemic control. With the findings of this study, providers can be reassured that the treatment of GE cancer does not negatively affect glycemic control among patients with DM. The risks of death and disease progression were greater in patients with GE cancer and DM than patients without DM. The mechanisms of this higher mortality rate in DM require further study.

\section{Summary points}

- The aim of the present case-control retrospective study was to evaluate the effect of diabetes mellitus (DM) on survival of patients with gastric or esophageal (GE) cancer and to determine whether GE cancer affects diabetes control.

- From an institutional registry, 184 patients with GE cancer were identified and grouped by the presence $(n=92)$ or absence $(n=92)$ of DM.

- The groups were matched for sex, age and year of GE cancer diagnosis.

- GE cancer did not affect glycemic control, and risks of death and disease progression were greater in patients with DM than patients without DM.

\section{Author contributions}

All the authors contributed equally to the conception and design of the study, data acquisition, analysis and manuscript creation.

Financial \& competing interests disclosure

The authors have no relevant affiliations or financial involvement with any organization or entity with a financial interest in or financial conflict with the subject matter or materials discussed in the manuscript. This includes employment, consultancies, honoraria, stock ownership or options, expert testimony, grants or patents received or pending, or royalties.

No writing assistance was utilized in the production of this manuscript. 
Ethical conduct of research

The authors state that they have obtained appropriate institutional review board approval or have followed the principles outlined in the Declaration of Helsinki for all human or animal experimental investigations.

Open access

This work is licensed under the Attribution-NonCommercial-NoDerivatives 4.0 Unported License. To view a copy of this license, visit http://creativecommons.org/licenses/by-nc-nd/4.0/

\section{References}

Papers of special note have been highlighted as: $\bullet$ of interest; $\bullet \bullet$ of considerable interest

1. American Cancer Society (2018). www.cancer.org/

2. Dixon JL, Copeland LA, Zeber JE et al. Association between diabetes and esophageal cancer, independent of obesity, in the United States Veterans Affairs population. Dis. Esophagus 29(7), 747-751 (2016).

3. Lauby-Secretan B, Scoccianti C, Loomis D et al. Body fatness and cancer - viewpoint of the IARC working group. N. Engl. J. Med. 375(8), 794-798 (2016).

4. Ogurtsova K, da Rocha Fernandes JD, Huang Y et al. IDF diabetes atlas: global estimates for the prevalence of diabetes for 2015 and 2040. Diabetes Res. Clin. Pract. 128, 40-50 (2017).

5. Tsai MS, Wang YC, Kao YH, Jeng LB, Kao CH. Preexisting diabetes and risks of morbidity and mortality after gastrectomy for gastric cancer: a nationwide database study. Medicine 94(37), e1467 (2015).

6. Miao ZF, Xu H, Xu YY et al. Diabetes mellitus and the risk of gastric cancer: a meta-analysis of cohort studies. Oncotarget 8(27), 44881-44892 (2017).

- Evaluates the evidence with respect to the relationship between diabetes mellitus (DM) and gastric cancer incidence or mortality.

7. Xu B, Zhou X, Li X, Liu C, Yang C. Diabetes mellitus carries a risk of esophageal cancer: a meta-analysis. Medicine 96(35), e7944 (2017).

- Via meta-analysis, the aim of this manuscript was to investigate the association between DM and esophageal cancer.

8. Huang W, Ren H, Ben Q, Cai Q, Zhu W, Li Z. Risk of esophageal cancer in diabetes mellitus: a meta-analysis of observational studies. Cancer Causes Control 23(2), 263-272 (2012).

9. de Jong R, Peeters P, Burden AM et al. Gastrointestinal cancer incidence in Type 2 diabetes mellitus; results from a large population-based cohort study in the UK. Cancer Epidemiol. 54, 104-111 (2018).

- The aim of this study was the determination of incidence rates of gastrointestinal (GI) cancers in patients with and without Type II DM in the UK.

10. Tseng CH, Tseng FH. Diabetes and gastric cancer: the potential links. World J. Gastroenterol. 20(7), 1701-1711 (2014).

11. Karlin NJ, Dueck AC, Reddy SKN, Verona PM, Cook CB. Implications of breast cancer with diabetes mellitus on patient outcomes and care. Diabetes Manag. 4(5), 411-419 (2014).

12. Karlin NJ, Amin SB, Verona PM, Kosiorek HE, Cook CB. Co-existing prostate cancer and diabetes mellitus: implications for patient outcomes and care. Endocr. Pract. 23(7), 816-821 (2017).

13. Karlin NJ, Amin SB, Buras MR, Kosiorek HE, Verona PM, Cook CB. Patient outcomes from lung cancer and diabetes mellitus: a matched case-control study. Future Sci. OA 4(1), FSO248 (2018).

14. Karlin NJ, Amin SB, Kosiorek HE, Buras MR, Verona PM, Cook CB. Survival and glycemic control in patients with colorectal cancer and diabetes mellitus. Future Sci. OA 4(9), FSO335 (2018).

15. Karlin NJ, Amin SB, Kosiorek HE, Buras MR, Verona PM, Cook CB. Survival and glycemic control outcomes among patients with coexisting pancreatic cancer and diabetes mellitus. Future Sci. OA 4(4), FSO291 (2018).

16. Faries D, Obenchain RL, Leon AC, Haro JM. Analysis of Observational Health Care Data Using SAS. SAS Institute, Inc., NC, USA (2010).

17. Tseng $\mathrm{CH}$. Diabetes conveys a higher risk of gastric cancer mortality despite an age-standardised decreasing trend in the general population in Taiwan. Gut 60(6), 774-779 (2011).

18. Hu D, Peng F, Lin X et al. Preoperative metabolic syndrome is predictive of significant gastric cancer mortality after gastrectomy: the Fujian Prospective Investigation of Cancer (FIESTA) study. EBioMedicine 15, 73-80 (2017).

19. Hu D, Peng F, Lin X et al. The elevated preoperative fasting blood glucose predicts a poor prognosis in patients with esophageal squamous cell carcinoma: the Fujian prospective investigation of cancer (FIESTA) study. Oncotarget 7(40), 65247-65256 (2016).

20. He LR, Qiao W, Liao ZX et al. Impact of comorbidities and use of common medications on cancer and non-cancer specific survival in esophageal carcinoma. BMC Cancer 15, 1095 (2015).

-. The objective of this manuscript was to investigate the co-morbidities and/or medications that may have an impact on esophageal cancer survival. 
21. Yao W, Meng Y, Lu M et al. Impact of Type 2 diabetes mellitus on short-term and long-term outcomes of patients with esophageal squamous cell cancer undergoing resection: a propensity score analysis. Cancer Commun. 38(1), 14 (2018).

22. van de Poll-Franse LV, Houterman S, Janssen-Heijnen ML, Dercksen MW, Coebergh JW, Haak HR. Less aggressive treatment and worse overall survival in cancer patients with diabetes: a large population based analysis. Int. J. Cancer 120(9), 1986-1992 (2007).

-. The aim of this manuscript was to examine DM prevalence among newly diagnosed cancer patients and to investigate how DM impacts stage at diagnosis, treatment and overall survival.

23. Salem ME, Puccini A, Xiu J et al. Comparative molecular analyses of esophageal squamous cell carcinoma, esophageal adenocarcinoma, and gastric adenocarcinoma. Oncologist 23(11), 1319-1327 (2018).

-. The goal of this manuscript was to compare molecular characteristics of esophageal squamous cell cancer, esophageal adenocarcinoma and gastric adenocarcinoma. 